these levels differ over an exacerbation time course. We sought to investigate the effects of Haemophilius influenzae cell-interaction upon airway inflammation and whether the levels of $H$. influenzae bacteria and cell-dissociated bacteria differ over an exacerbation time course.

Methods Cell differential counts were carried out on sputum samples as per standard protocol. Bacterial DNA was extracted and H.influenzae was quantified using qPCR from the sputum plug (contains cell-associated and dissociated bacteria) and the sputum cell-free supernatant (cell-dissociated bacteria only). Inflammatory mediators (IL- $1 \alpha$, TNF- $\alpha$, IL- 8 and neutrophil elastase (NE)) were measured in the sputum supernatant using commercial assays.

Results 63 patients (77\% male; average age of 69 (45-88); $\mathrm{FEV}_{1}$ percentage predicted of $53 \%$; mean percentage neutrophil count in sputum of $65 \%$ ) at stable state were analysed. Levels of $H$. influenzae in the supernatant only correlated with the sputum total cell count $(\mathrm{r}=0.38 ; \mathrm{p}=0.03)$. Levels of $H$. influenzae in the plug correlated with inflammatory mediators (sputum neutrophil percentage $\mathrm{r}=0.42, \mathrm{p}=0.01$; sputum macrophage percentage $\mathrm{r}=-0.35, \mathrm{p}=0.04 ; \mathrm{IL}-1 \alpha \mathrm{r}=0.36$, $\mathrm{p}=0.03 ; \mathrm{IL}-8 \mathrm{r}=0.49, \mathrm{p}<0.01 ; \mathrm{NE} \mathrm{r}=0.40, \mathrm{p}=0.02)$. The exacerbation time course in 10 paired COPD subjects was examined. There was no significant difference in $H$. influenzae levels in the plug $(p=0.89)$ (figure $1 \mathrm{~A})$. However, there was a significant increase in levels in the supernatant over the exacerbation time course $(p=0.05)$ (figure 1B).

Conclusion $H$. influenzae levels in the sputum plug appear to have much more of an effect on airway inflammation than levels of cell-dissociated $H$. influenzae suggesting that cell-associated bacteria may be a driver of airway inflammation in COPD. Further investigation into this highly complicated relationship needs to be conducted.

\section{S117 THE EFFECT OF CIGARETTE AND ELECTRONIC CIGARETTE VAPOUR ON BACTERIA IN CHRONIC LUNG INFECTION}

${ }^{1} \mathrm{DF}$ Gilpin, ${ }^{1} \mathrm{KA}$ McGown, ${ }^{1} \mathrm{~K}$ Gallagher, ${ }^{1} \mathrm{~J}$ Bengoechea, ${ }^{2} \mathrm{JS}$ Elborn, ${ }^{1} \mathrm{MM}$ Tunney. ${ }^{1}$ Queen's University, Belfast, UK; ${ }^{2}$ Imperial College, London, UK

\subsection{6/thoraxjnl-2017-210983.123}

Introduction Cigarette smoke is a risk factor for the development of several chronic lung diseases, e.g., Chronic Obstructive Respiratory Disease (COPD) and bronchiectasis. This study aims to determine the effect of cigarette smoke and electronic cigarette vapour, on key bacteria involved in the progression and exacerbation of chronic lung disease.

Methods Cigarette smoke extract (CSE) and electronic cigarette vapour extract (ECVE) were prepared using a modified syringe driver apparatus and bubbling through appropriate growth media. Reference isolates [Haemophilus influenzae, ATCC49766 (HI), Streptococcus pneumoniae ATCC49619 (SP), Staphylococcus aureus ATCC29213 (SA) and Pseudomonas aeruginosa ATCC 27853 (PA)] were grown in broth +/CSE or ECVE. Biofilm formation and survival in the Galleria mellonella infection model, following bacterial exposure to CSE/ECVE were determined. Levels of IL- 8 and TNF $\alpha$ secretion from A549 cells, following infection by bacteria+/-CSE/ ECVE and addition of cell-signalling inhibitors, were measured by ELISA.
Results A trend towards increased biofilm formation following exposure to either CSE or ECVE was observed, which reached statistical significance with $\mathrm{SP}$ and $\mathrm{PA}+\mathrm{CSE}$ $(\mathrm{p}=0.0047$ and 0.0043 , respectively) and SA+ECVE $(\mathrm{p}<0.001)$. There was decreased survival of Galleria mellonella following infection with bacteria $+\mathrm{CSE} / \mathrm{ECVE}$ vs. bacteria only, suggestive of increased virulence; this was statistically significant in the case of $\mathrm{HI}(p=0.016)$ and PA $(p=0.0005)$ + CSE. Statistically significant increases in IL-8 secretion in A549 cells were observed for $\mathrm{HI}$ and PA +CSE and SA + ECVE, and in TNFo, with SP and SA+ECVE. However, a clear trend towards increases in both cytokines following CSE/ ECVE exposure was evident, with little difference observed between CSE/ECVE. Addition of pathway inhibitors following A549 cell infection with bacteria+CSE/ECVE resulted in a decrease in IL-8 or TNF $\alpha$ via the same pathways as with bacteria alone.

Conclusion Exposure of bacteria involved in the pathogenesis of chronic lung infection to both CSE and EVCE resulted in increased virulence, biofilm formation, and inflammation, and may contribute to establishment of chronic infection and persistence. Further work is required to determine the clinical effects of ECVE.

\section{Of mice and men}

\section{S118 ELK1 GENE DELETION LEADS TO SPONTANEOUS EARLY FIBROTIC CHANGES IN THE AGEING LUNG}

${ }^{1}$ AN Habgood, 'E Hampson, ${ }^{1} \mathrm{~J}$ Cairns, ${ }^{1} \mathrm{AE}$ John, ${ }^{2} \mathrm{~S}$ Alberti, ${ }^{2} \mathrm{~A}$ Nordheim, ${ }^{1} \mathrm{G}$ Jenkins, ${ }^{1}$ AL Tatler. 'Nottingham Respiratory Research Unit, University of Nottingham, Nottingham, UK; ${ }^{2}$ Interfaculty Institute of Cell Biology, Tübingen University, Tübingen, Germany

\subsection{6/thoraxjnl-2017-210983.124}

Rationale Idiopathic pulmonary fibrosis is a chronic fibroproliferative disease with a median survival of approximately 3 years. $\alpha v \beta 6$ integrins are upregulated in lung fibrosis and are associated with increased activation of the profibrotic cytokine TGF- $\beta$. The transcription factor Elk1 can repress gene expression of $\beta 6$. We therefore hypothesise that animals lacking functional Elk1 $\left(E l k 1^{-/ 0}\right)$ will develop age related pulmonary fibrosis.

Methods Elk1 knock-out $\left(E l k 1^{-/ 0}\right)$ and wild-type $\left(E l k 1^{+/ 0}\right)$ mice were allowed to age for 365 days. At 365 days old mice were sacrificed and their lungs harvested for evaluation of collagen gene expression, lung hydroxyproline concentration and histological assessment.

Results Lungs were extracted and lung wet weights were measured in Elk1 $1^{-/ 0}$ mice and wild-type $\left(E l k 1^{+/ 0}\right)$ controls and no significant difference between the two genotypes was shown (175.7 mg, $161.8 \mathrm{mg}$ respectively $\mathrm{n}=6-8$ ). However, assessment of total lung hydroxyproline established that there was significantly more hydroxyproline in Elk1 $1^{-/ 0}$ mice compared with $E l k 1^{+/ 0}$ controls $(852.3,758.4 \mu \mathrm{g} /$ lung set, respectively, $\mathrm{n}=5-8, \mathrm{p}=0.0346)$. Assessment of Masson's trichrome stained $E l k 1^{-/ 0}$ lung tissue sections found a small number of fibrotic lesions were present. Furthermore, there was a trend towards increased alveolar wall median thickness in Elk1 $1^{-10}$ mice compared with $E l k 1^{+/ 0}$ animals (5.94 vs $5.56 \mu \mathrm{m}$ respectively). In a small number of 12 week old mice we identified a trend towards increased $\alpha$-smooth muscle actin ( $\alpha \mathrm{SMA}$ ) mRNA expression in the lungs of $E l k 1^{-/ 0}$ mice compared with 
Elk1 $1^{+/ 0}$ controls $(9.40,2.24$ median relative expression, respectively $n=3$ ). We therefore performed immunohistochemical staining for $\alpha \mathrm{SMA}$ in the lungs of mice aged to 1 year and demonstrated visible increases in expression of $\alpha \mathrm{SMA}$ in the alveolar epithelium of $E l k 1^{-/ 0}$ mice but not in $E l k 1^{+/ 0}$ controls.

Conclusion These data suggest that Elk1 gene deletion result in age-related early fibrotic changes associated with the development of pulmonary fibrosis.

\section{S119 MAPPING MOUSE MODELS OF SEVERE ASTHMA ONTO HUMAN DISEASE}

${ }^{1} \mathrm{~K}$ Kazi, ${ }^{2} \mathrm{IM}$ Adcock, ${ }^{3} \mathrm{~S}$ Pavlidis. ${ }^{1}$ Imperial College London, London, UK; ${ }^{2}$ National Heart and Lung Institute, London, UK; ${ }^{3}$ Data Science Institute, London, UK

\subsection{6/thoraxjnl-2017-210983.125}

Introduction Severe asthma represents a significant unmet need in terms of therapeutics. Drug development in asthma has been slow and expensive and one of the reasons for this is that positive findings for drugs tested in preclinical models have not readily translated into successful therapies in man.

Aims and Objectives We sought to improve the predictive power of existing models of asthma by using novel bioinformatics techniques to align these models with subsets of human asthma.

Methods We applied differential gene expression analysis to transcriptomic data from whole lung samples of 6 murine models of asthma and oxidative stress to produce gene signatures that represented each model. These signatures were then used to calculate enrichment scores (ESs) for transcriptomic data from bronchial biopsies taken from 81 asthmatic and 26 healthy subjects from the U -BIOPRED cohort using gene set variation analysis. These ESs were taken as an index of similarity between each model and each patient and were used to drive further analyses using topological data analysis and goodness of fit modelling.

Results We found that no single mouse model was aligned well with all asthmatics. We identified three clusters of patients who were represented to varying degrees by different mouse models and who displayed clinical features that aligned well with phenotypes of asthmatics identified previously by clustering analyses based upon clinical features and biological markers. Patients in cluster $\chi$ were defined by neutrophilic sputum, later onset of disease, higher incidence of sinusitis, more frequent exacerbations and more airflow limitation. Patients in cluster Y1 showed significantly lower sputum neutrophils, a trend towards higher sputum eosinophils, a significantly later onset of airways disease and a trend towards higher BMI. Patients in cluster Y2 showed a significantly higher percentage of neutrophils in the blood, a trend towards increased sputum lymphocytes and were more likely to identify aspirin as a trigger $(\mathrm{p}=0.06)$.

Conclusion Our evidence supports the assertion that it is possible, on a transcriptional level, to align mouse models of asthma to subsets of human asthma and that doing so may have significant implications for the expedience of drug development in asthma.

\section{S120 GENE THERAPY FOR PULMONARY ALVEOLAR PROTEINOSIS}

${ }^{1} \mathrm{~N}$ Atsumi, ${ }^{1} \mathrm{~A}$ Pilou, ${ }^{2}$ Pringle, ${ }^{2} \mathrm{RC}$ Ashworth, ${ }^{3} \mathrm{C}$ Meng, ${ }^{3} \mathrm{M}$ Chan, ${ }^{2} \mathrm{DR}$ Gill, ${ }^{2} \mathrm{~S}$ Hyde, ${ }^{4} \mathrm{C}$ Morgan, ${ }^{1} \mathrm{EWFW}$ Alton, ${ }^{3} \mathrm{U}$ Griesenbach. ${ }^{1}$ Department of Gene Therapy, NHLI, Imperial College London and UK Gene Therapy Consortium, London, UK; ${ }^{2}$ Radcliffe Department of Medicine, Oxford University and Gene Therapy Consortium, London, UK; ${ }^{3}$ Department of Gene Therapy, NHLI, Imperial College London and Raddliffe Department of Medicine, Oxford University, London, UK; ${ }^{4}$ Royal Brompton Hospital, London, UK

\subsection{6/thoraxjnl-2017-210983.126}

Introduction Pulmonary alveolar proteinosis (PAP) is characterised by accumulation of surfactant in the terminal airways. Granulocyte-Macrophage Colony-Stimulating-Factor (GM-CSF) stimulates alveolar macrophages to clear surfactant. The presence of GM-CSF autoantibodies in autoimmune PAP (aPAP) leads to surfactant build-up and impaired gas exchange. This causes respiratory symptoms and can ultimately be fatal due to hypoxaemic respiratory failure. We hypothesise that lentivirus-mediated gene transfer of GM-CSF may be suitable to treat aPAP and propose to assess efficacy of GM-CSF gene transfer in GM-CSF knockout mice, which recapitulate aPAP lung disease. The murine GM-CSF (mGM-CSF) cDNA was cloned into a lentiviral vector, which was pseudotyped with the $\mathrm{F}$ and $\mathrm{HN}$ proteins from Sendai virus to enable efficient lung transduction (rSIV.F/HN-mGM-CSF).

Methods and Results To confirm if the vector produces mGMCSF we first transduced A549 cells with multiplicity of infection (MOI) of $0.1-100$ ( $n=6 /$ group). 48 hours after transduction dose-related mGM-CSF expression was confirmed in the medium. We next assessed whether the mGM-CSF produced after gene transfer was biologically active by comparing the proliferation rate of FDC-P1 cells, a mGM-CSF-dependent mouse myeloid progenitor cell line, in the presence of gene therapy- produced mGM-CSF $(0.001-10 \mathrm{ng} / \mathrm{ml})$ and purchased recombinant $\mathrm{mGM}-\mathrm{CSF}$ protein $(\mathrm{n}=6 /$ group). The dose-related proliferation rates in both conditions were similar (figure 1ss). In preliminary experiments, we next assessed whether gene transfer led to GM-CSF production in vivo. rSIV.F/HN-mGMCSF (1e7 transduction units (TU)/mouse) was administered to wild-type mice by nasal "sniffing". Control mice remained untransduced ( $n=3 /$ group). mGM-CSF levels were quantified in lung tissue homogenate and broncho-alveolar lavage fluid (BALF) 14 days after gene transfer. mGM-CSF levels in untreated mice were below the limit of detection of the ELISA, but high levels of mGM-CFS were detectable in lung tissue (median 825 (range 460-3790) pg/mg) and BALF (median: 3330 (range 2307-7958) pg/ml).

Conclusion rSIV.F/HN-mGM-CSF produced mGM-CSF in vitro and in vivo. The biological function of the protein was confirmed in vitro and evaluation of mGM-CSF gene transfer efficacy in murine aPAP model is ongoing. 